\title{
DESARROLLO DE HARNERO VIBRATORIO
}

\author{
Ernesto Ponce L. ${ }^{1}$ René Cortés F. ${ }^{1} \quad$ Claudio Valdés R. ${ }^{1}$ \\ Recibido el 30 de julio de 2003, aceptada el 30 de diciembre de 2003
}

\begin{abstract}
RESUMEN
Se presenta el desarrollo del cálculo de un harnero vibratorio terciario de pendiente variable, para ser construido en la Maestranza de Chuquicamata. La relevancia de realizar este proyecto radica en la necesidad de satisfacer los nuevos requerimientos de producción y acortar los tiempos de mantenimiento empleando nuevos conceptos de diseño (como variación de algunos parámetros de operación en terreno) y componentes de materiales avanzados de intercambio rápido. Como elemento de verificación del diseño, se construyó un prototipo de dimensiones mas reducidas, empleando cálculos para un equipo real, sin utilizar reglas de reducción de escala, por no disponer de esa información para equipos de minería . El bajo costo respecto a los equivalentes ofertados en el extranjero y la demostración de las bondades de su funcionamiento, indican que es rentable la fabricación de estas maquinarias en el país.
\end{abstract}

Palabras Claves: Harnero vibratorio, tamiz pendiente variable

\begin{abstract}
This paper shows the development of a calculation of a vibrating screen with variable slope, to be built in Chuquicamata. This project is relevant because it helps satisfy the new production demand and reduces maintenance time. It employs new design concepts and uses advanced materials of fast interchange. As comparison method, a reduced model was built and tested. The calculations were based on a machine of reduced dimensions because it was not possible to use those scale rules for mining equipment. The low manufacturing cost of the designed screen compared with the product supplied abroad, as well as the model good performance, indicates that the manufacture of these machines in Chile is profitable.
\end{abstract}

Keywords: Vibrating screen, variable slot screen

\section{INTRODUCCIÓN}

Al año 2000 los harneros terciarios de la Planta de Chancado Terciario de Chuquicamata, eran marca Tyler, modelo Ty-Rock [1] mas conocidos como F-600, pueden definirse como una máquina vibradora de 4 cojinetes con un eje excéntrico doble, que produce un movimiento de carrera en círculo. El cuerpo del vibrador y el eje que soporta los brazos laterales están aislados de la estructura base por amortiguadores de goma. Cuenta con dos mallas de alambrón para cribado, de abertura 19x123 mm la primera y 13,5x $99 \mathrm{~mm}$ la segunda. Las principales fallas eran de soltura de correas de transmisión, fallas eléctricas, roturas de brazo soporte de amortiguadores; desgaste de mallas, rodamientos y amortiguadores, entre otros. Las mallas debían voltearse cada semana y ser cambiadas cada 15 días, este solo ítem obligaba a planificar detenciones semanales de la sección, lo que exigía buscar soluciones mas duraderas. Anualmente el tiempo de detención promedio era de 6737 horas, por lo que las pérdidas de disponibilidad eran del $6 \%$ de la Planta de Chancado. Como reflejo de estas fallas, la detención de un harnero repercutía en su gemelo y el chancador terciario asociado, porque cuando se detiene uno, todo el flujo se traspasa al otro [2]. La capacidad se veía colapsada, además de pasar un alto porcentaje de material fino al triturador de cono, con el consiguiente desgaste de su coraza y aumentando la probabilidad de atascamiento. Resumiendo los efectos, se tenía que anualmente dejaban de procesarse 296428 toneladas de mineral, al año 2000 eran pérdidas de US \$303165 mas US \$ 593272 por gastos de mantenimiento, no se incluyen los indirectos como fallas en los chancadores. Dado que debían reemplazarse 20 equipos, ubicados en lugares con espacios muy restringidos y en altura, se decidió la

\footnotetext{
${ }^{1}$ Universidad de Tarapacá - CODELCO, Departamento de Ingeniería Mecánica, Casilla 6 D, Arica Chile, Fono 58-205293 FAX 58-205281, eponce@uta.cl
} 
sustitución por 10 nuevos, con dimensiones apropiadas . Existiendo muchos modelos y fabricantes, además de muchos parámetros. Debía seleccionarse un tipo de diseño avanzado, de probada eficacia, no para copiarlo, sino para comparar sus ventajas respecto a los modelos mas convencionales y considerar las nuevas ideas, ver Fig.1. También debía tenerse en cuenta la composición química del mineral (por su potencial agresividad con los materiales empleados); la granulometría, la gravedad específica, la densidad, su contenido de humedad (para decidir si se trata de harneado seco, húmedo o mojado); las características abrasivas, corrosivas, ángulo de fricción, ángulo de deslizamiento y forma de la partícula entre otros [3], [4]. Otras consideraciones debían incluir los prejuicios y las inclinaciones de los operarios, esto debe recibir una cuidadosa atención, así como los espacios para mantención y cambio de componentes. Debía pensarse en la standarización de repuestos; en la dirección de rotación de los vibradores excéntricos; el ángulo de inclinación de los tamices, la selección del golpe adecuado (golpe = amplitud x2);la velocidad de rotación del sistema vibratorio; abertura de las mallas; profundidad del lecho y flujo del material [1], [5]. Como filosofía de diseño se pensó en hacer una unidad donde se pudiera variar la fuerza de excitación, la suspensión y el ángulo, todo dentro de un rango razonable. De esta manera sería mas fácil adaptar el equipo a las condiciones de trabajo requeridas y controlar problemas no considerados. En la parte relacionada con la resistencia de los materiales debía analizarse en todas las piezas el efecto de fatiga y concentración de tensiones [6], [7], [8].

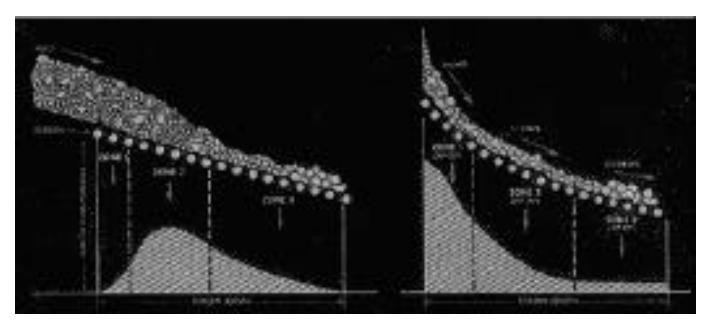

Fig.1.- Comparación entre harnero clásico y uno de

\section{DESARROLLO}

Se realizaron los cálculos de un harnero de pendiente variable [9], capaz de satisfacer los requerimientos de alimentación de un chancador terciario. Luego estos un modelo a escala reducida .Como se carecía de la información de leyes de escala para equipos de minería, el cálculo del modelo se realizó como si se fuese a pendiente variable cálculos teóricos serían verificados con los resultados de

comportar en condiciones reales de trabajo. Se decidió utilizar dos bandejas de tamizado (decks) a semejanza de los antiguos F-600. Pero, a diferencia de estos últimos, ellas tienen una pendiente alta en la entrada, que se reduce luego en el centro y disminuye aún más en la salida, ver Fig.4. No hay una variación continua, por las dificultades constructivas que eso traería. El primer análisis debe realizarse sobre los tamaños de las partículas y su geometría: los efectos son diferentes si son redondeadas o si tienen aristas. El ángulo de fricción interna proporciona la tangente trigonométrica, que es el coeficiente de rozamiento del material. El ángulo de reposo se produce cuando se le deposita sobre una superficie horizontal, aquí la compactación de las partículas interiores genera un cambio en la fricción, con lo cual este ángulo es menor que el de fricción interna. Otro cálculo importante es el área del tamiz, una buena proporción es que el largo sea el doble que el ancho, aunque también se recomiendan otras relaciones[3], [4], [5]. Una vez determinado el flujo del harnero en toneladas cortas / hora, Allis Chalmers [3] recomienda la siguiente formulación:

Área en pies2 $=$ Flujo/(C* $\left.\mathrm{M}^{*} \mathrm{~K}^{*} \mathrm{Q} 1 * \mathrm{Q} 2 * \mathrm{Q} 3 * \mathrm{Q} 4 * \mathrm{Q} 5\right)$

Donde:

$\mathrm{C}=$ factor relacionado con la abertura del tamiz (en pulgadas)

$\mathrm{M}=$ factor relacionado con el $\%$ de material mayor que la abertura del tamiz

$\mathrm{K}=$ factor relacionado al $\%$ de material que tiene la mitad de la abertura del primer tamiz

Q1 = Factor de la densidad volumétrica

$\mathrm{Q} 2=$ Factor del tipo de abertura

$\mathrm{Q} 3=$ Factor de forma de la partícula

Q4= Factor de tamaño de la abertura

Q5= Factor de humedad superficial

Otros fabricantes, por ejemplo la Asociación de Fabricantes de Harneros Vibratorios, VSMA, emplean formulaciones parecidas, incluyendo las tablas o gráficas para seleccionar los factores. La profundidad del lecho es importante y se determina como :

ELD $=$ espesor del lecho del material en la descarga $=$ $\mathrm{O} * \mathrm{D} /(5 \mathrm{~V} * \mathrm{~W})$

Donde:

$\mathrm{O}=$ material con sobretamaño en toneladas cortas/hora

$\mathrm{D}=$ densidad en toneladascortas/pie3 
$\mathrm{V}=$ velocidad del material sobre el harnero en pies/minuto

$\mathrm{W}=$ ancho de la superficie de cribado en pies

Debe cumplirse que el espesor del lecho sea menor que cuatro veces la abertura del tamiz [3], [4], [5].

La eficiencia de remoción de material bajo tamaño debe ser probada. Si se considera que el sobretamaño es el producto, el harnero debería ser capaz de sacar todo el material de tamaño menor a la abertura, pero ningún equipo es comercialmente capaz de hacer esto. La eficiencia de material removido de bajo tamaño en $\%$, sería 100 menos el \% de bajo tamaño contenido en el que sale junto al de sobre tamaño. La eficiencia en sobre tamaño es \% de sobre tamaño en la alimentación por $100 /(\%$ que pasa a la descarga del deck). También existen otras formulaciones para determinar eficiencias. Para harneado seco la selección del vibrador debe ser de dos o cuatro rodamientos (golpe positivo), ambos tienen un buen comportamiento pero la unidad de cuatro arranca y se detiene mas suave, ver Fig.2. Se denomina golpe a dos veces la amplitud de oscilación. La dirección de los vibradores puede ser a favor o contra flujo del material. Los proveedores recomiendan un ángulo de variación de la inclinación de dos grados de la vibración respecto del tamiz. También los fabricantes proporcionan tablas donde recomiendan la longitud del golpe en pulgadas en función del rango de pendiente del deck; la velocidad nominal en rpm del vibrador y el rango de abertura del tamiz, en malla o pulgadas.

\section{SISTEMA VIBRADOR}

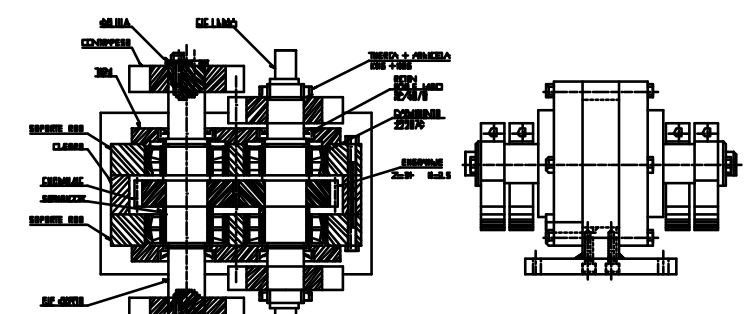

Cajo exateckra en corte

Cojo excitadora. Elevación

Fig. 2.- Vibrador de masas excéntricas

Es el corazón del equipo y genera la fuerza de excitación que da el movimiento oscilatorio. En este trabajo se han elegido las masas de giro contrapuesto, para generar un movimiento lineal paralelo a la criba (o aproximadamente). Esto facilita la clasificación para este tipo de material que tiene una pendiente variable en el deck. Se hace notar que en los modelos horizontales se prefiere el movimiento lineal y en los inclinados el elíptico o circular. En nuestro caso el primer sector no requiere demasiada vibración para lograr la estratificación por tener una gran pendiente y alcanzar gran velocidad (Fig.1). Hacia el final del segundo sector y en la zona de descarga hay una superficie semi plana que necesita fuerza de excitación para mover el material hacia la salida, ver Fig.4. Como el sistema es flexible, si no resultare bien se puede modificar a un movimiento elíptico con sólo cambiar de posición los engranajes. Ver Fig. 2. La fuerza del vibrador se obtiene de relaciones de los fabricantes. Esta fuerza de excitación proporcionada por los contrapesos es igual a la masa excéntrica por el radio de excentricidad por la velocidad angular al cuadrado. Las características del motor se obtienen a partir del torque requerido, que puede calcularse como la aceleración angular por el momento de inercia de las masas del sistema de excitación. La aceleración angular se obtiene de la velocidad de giro del vibrador dividido por el tiempo que demora el sistema en alcanzar dicha velocidad, el que medido en terreno dio una media de 5 segundos. Conocidas las rpm del sistema (que deben ser menores a 1200 por recomendación de los proveedores), se determina el torque. La potencia de accionamiento es el torque multiplicado por la velocidad de giro del sistema. Como es un diseño flexible, en el transcurso de las pruebas se pueden variar los contrapesos (Fig.3), luego el momento de inercia también se puede modificar. La transmisión se realiza por engranajes rectos que no producen cargas axiales, esto es muy importante porque los rodamientos estarán sometidos a cargas de impacto, fatiga, temperatura, contaminación entre otros; también hay que pensar en los sellos que no deben permitir escapes de lubricante para no dejar seca la transmisión ni contaminar el producto. Los rodamientos empleados fueron de rodillos a rótulas de vida calculada en 20000 horas y factor de seguridad 6 en la capacidad de carga dinámica (Fig.2). Esto puede ser motivo de polémica, pero su costo es ínfimo frente al de una detención no programada. Los ejes se calcularon para acero AISI 4140 tratado térmicamente y normalizado a 900 grados C , mediante el código ASME [6], para vida indefinida y confiabilidad 99,99. La caja se hizo de placas apernadas para evitar las distorsiones producidas por la soldadura, con los consiguientes desalineamientos de los ejes. El espesor para alojamientos de rodamientos y sellos fue mas que suficiente para dar rigidez al sistema como un todo monolítico.

\section{ESTRUCTURA}

La estructura se diseñó en acero ASTM A 36 por tener 
menos pasadas por el laminador y por consiguiente, menos tensiones residuales por deformación. Para evitar estas tensiones impresas se excluyeron soldaduras, siendo casi todas las uniones con pernos de alta calidad y resistencia.

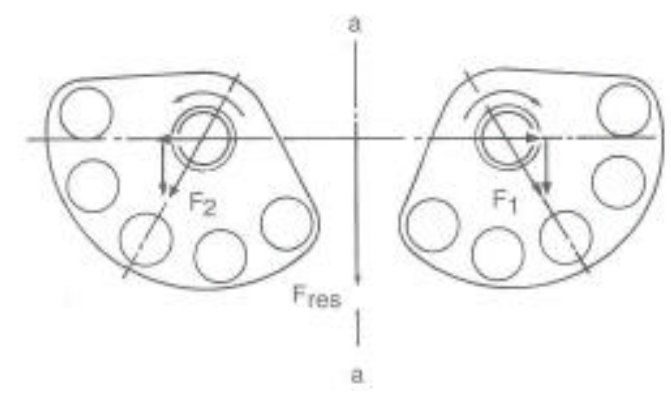

Fig. 3.- Masas de contrapesos opuestos

En los apoyos del sistema de suspensión las soldaduras eran inevitables, luego en estos lugares se especificó un alivio de tensiones, para evitar fallas por fatiga . Por motivos de tamaño no se incluyeron pernos patente HUCK, pero se fabricaron equivalentes. En los costados se montaron rigidizadores para eliminar vibraciones en las planchas (Fig. 6) además se pusieron barras separadoras cuya función era darle una rigidez tridimensional a la estructura. Los espesores de material se calcularon por teoría de estructuras, pero incluyendo efecto de fatiga y un factor de servicio pesado. El cálculo de los pesos de las partes y momentos de inercia del conjunto se realizó con un programa computacional. Una entrada del material, plana y cubierta de goma proporcionaba un acceso amortiguado al material, aparte que el elastómero antiabrasivo evita el desgaste de la plancha desnuda. En los costados de las bandejas también se puso bandas de elastómeros.

\section{SISTEMA DE SUSPENSIÓN}

El bastidor va soportado sobre placas nervadas que sujetan los resortes verticales. Hay dos placas en la entrada que soportan la mayor carga y dos en la salida . Las de la entrada están a mayor altura, por razones de la pendiente. Los resortes descansan sobre un soporte que está aislado de la base mediante otros resortes verticales, que debilitan la transmisión de vibraciones al piso, adicionalmente se han montado en este sector cuatro pares de resortes inclinados para contrarrestar rotaciones angulares a lo largo del equipo, ver Fig. 5 y Fig. 6. Se plantean 3 ecuaciones diferenciales para el movimiento del harnero : Una en el eje vertical $X$, otra para el Y horizontal y una ecuación de rotación en el plano $\mathrm{X}-\mathrm{Y}$. El eje $\mathrm{Z}$ transversal no se considera por no tener utilidad directa en el harneado. Otras tres ecuaciones similares se plantean para el movimiento del soporte de la estructura [10], [11], que también va suspendido con resortes. Habría seis grados de libertad, introduciendo una solución particular que se deriva dos veces y se introduce en las ecuaciones generales, construyendo una matriz de seis por seis y resolviendo el determinante igualado a cero (mediante un software iterativo que permite determinar los modos de vibración principales no amortiguados, con el método de Stodola) se obtienen las seis frecuencias naturales del sistema, que en teoría deben evitarse para no entrar en resonancia. Las masas, centros de masas y momentos de inercia se calculan por AUTOCAD. Los resultados obtenidos indican que las frecuencias de oscilación del harnero son mucho mas altas que las naturales: 125 frente a $\operatorname{los} 15,3$ a 26,6 radianes/segundo.

Las fuerzas $\mathrm{G}$ de aceleración del equipo tienen gran influencia en la forma de vibrar y en la duración de los

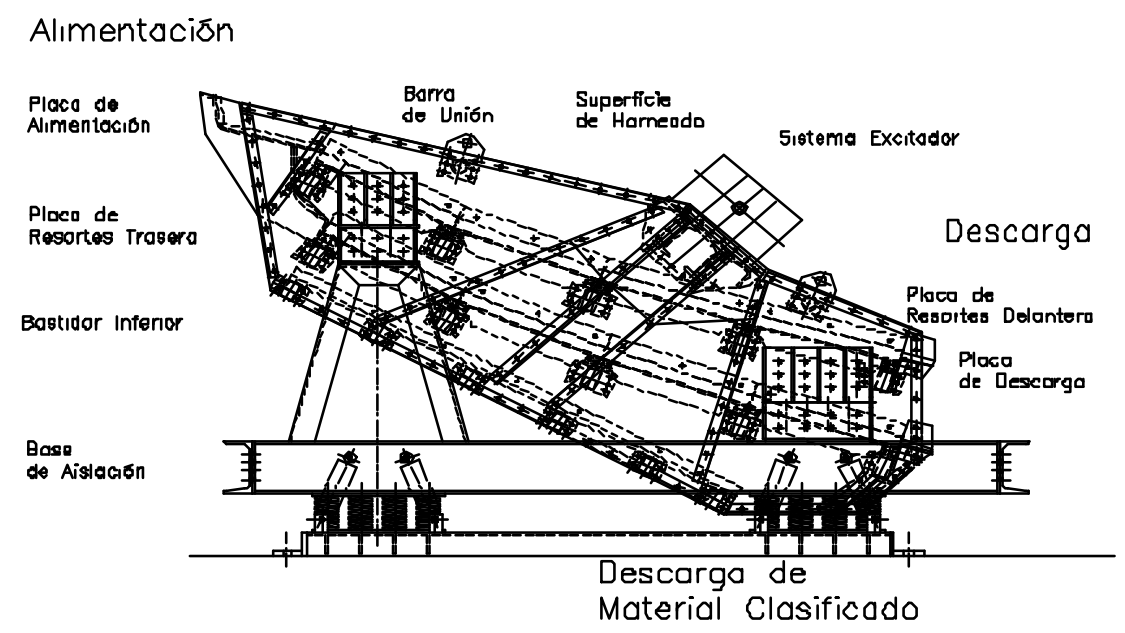

Fig. 4.- Estructura del Harnero 
Dependen del momento estático de los contrapesos, de las rpm y del peso total del harnero sin carga. Un fabricante la define como: rpm2 por 2 veces el momento estático de los contrapesos dividido por una constante y el peso del harnero sin carga. Para el caso del modelo desarrollado $G$ resultó 1,05 veces la aceleración de gravedad. La geometría de los resortes de sección circular se determina por las relaciones de Wahl, resistencia a la fatiga y duración indefinida [6]. El material fue acero ASTM A231 al cromo vanadio tratado térmicamente. Se emplearon además amortiguadores de goma para absorber vibraciones no deseadas.

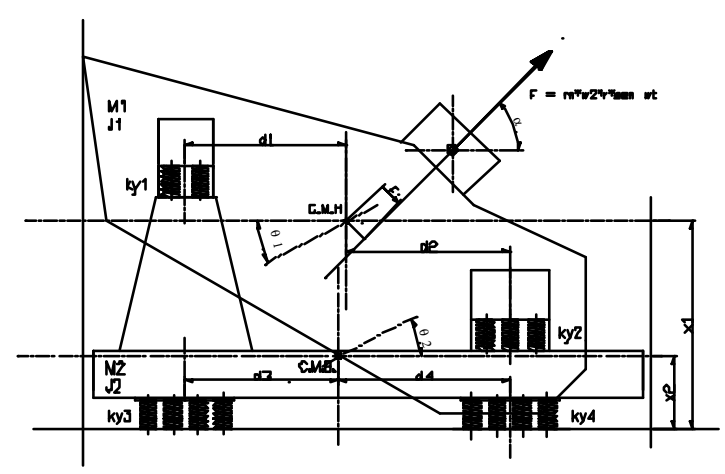

Fig. 5.- Suspensión

\section{MÓDULOS DE POLIURETANO}

Dentro de las superficies de cribado en los tipos de placas perforadas se incluyen estos módulos. Tienen hasta 5 veces mas duración que las mallas metálicas en aplicaciones de material seco y húmedo. Son elásticos y ceden bajo carga de impacto, pero no se rompen porque la absorben. Resisten temperaturas en rango de -35 a 176 grados $\mathrm{F}$ y resisten los solventes comunes. Se fabrican por moldeo a inyección en paneles standard múltiplos de un pie cuadrado( $1 \mathrm{X} 1,1 \mathrm{X} 2,1 \mathrm{X} 3$...). Al tener configuración modular se pueden reemplazar fácilmente en zonas de mayor desgaste y no en toda la superficie como en las mallas de metal. El montaje y desmontaje lo hace una sola persona empleando elementos de unión trabados (similares a pernos de plástico, pero que funcionan con una cuña interior que impide su despiece). Este tipo de material fue empleado en el prototipo.

\section{TRANSMISIÓN}

La transmisión original contemplaba dos motores montados en ambos costados del harnero, conectados directamente al eje mediante acoplamientos cardánicos o flexibles, esto da un diseño bien balanceado y sin los problemas propios de una conexión por correas, que entre otras dificultades incluye una importante carga radial y potenciales desalineamientos En el tema largamente discutido, primó el criterio de un solo motor conectado mediante correas trapezoidales a poleas de acero fundido (que tienen mayor duración frente a las de aluminio). Algunas mínimas dificultades presentadas en el prototipo dieron la razón a la primera alternativa.

\section{PRUEBAS}

El prototipo se hizo funcionar sin carga por varios días sin detenerse. También se hizo trabajar con carga alimentado desde una tolva. En ambos casos no presentó problemas, salvo una leve acción de frenado debido a variación de tensión de las correas trapezoidales descrita con anterioridad.

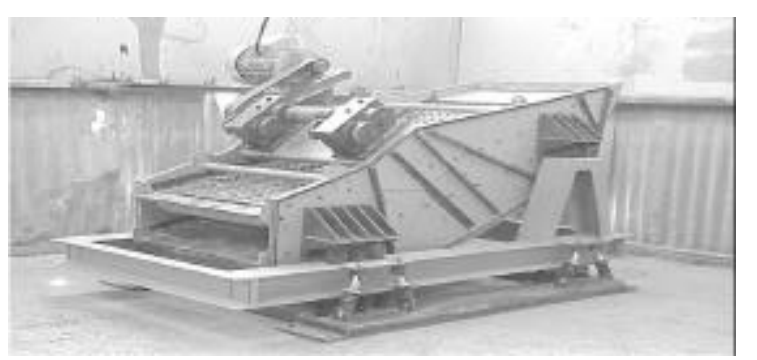

Fig. 6.- Perspectiva del harnero de pendiente variable: 35-20-10 grados

\section{CONCLUSIONES}

El desarrollo del prototipo permitió establecer que ciertos parámetros deben diseñarse con holgura, así hay una mayor flexibilidad en la operación. Se ha demostrado que es posible proyectar y construir algunos equipos de minería en el país con tecnología moderna. El beneficio económico de elaborar un harnero propio se traduce en una reducción de un $50 \%$ del costo de uno equivalente importado, con la ventaja de disponer de repuestos standard y el conocimiento para repararlos. Esto se puede proyectar a la exportación de estos harneros, no limitándonos a las materias primas, sino también a productos manufacturados.

\section{BIBLIOGRAFÍA}

[1] Tyler Industrial Products: "Manual de Funcionamiento , Mantención y Partes para Harnero Vibrado r Ty-Rock", USA, 1998.

[2] D. Romanini, R. Steigmaier; Informe Final Proyecto: "Continuidad de Marcha en Planta de 
Chancado Secundario-Terciario CODELCO Chuquicamata”, UTFSM ,Depto. Industrias, 1998.

[3] Allis Chalmes (Svedala); "Vibrating Screen, Theory and Selection", printed in USA, Allis Chalmes Co.

[4] C. Matthews; "Que Saber Sobre los Harneros", Publicaciones Mc Lean-Hunter, Reimpreso por Rocks Products, 1997.

[5] VSMA, "Vibrating Screen”, USA, 1998.

[6] V. Faires; "Diseño de Elementos de Máquinas", Ed. Uteha, primera edición en español, México 1990.

[7] R. Mott; "Diseño de Elementos de Máquinas", Edit. PHH, segunda edición, México, 1997.

[8] R. Norton; "Diseño de Máquinas", Editorial Pearson, Primera Edición, México, 1999.

[9] Nordberg; "Multi-Slope Banana Vibrating Screen Instruction Manual", 1999.

[10] R. Singeresu; "Mechanical Vibrations", Adison Wesley Publising Company, USA, 1996.

[11] W. Thomson; "Teoría de Vibraciones", Editorial PHH, Segunda Edición, México, 1996. 